\title{
Spin asymmetry in electron impact ionization of caesium
}

\author{
G Baum, B Granitzat, L Grau, B Leuer, W Raith, K Rott, \\ M Tondera and B Witthuhn \\ Fakultät für Physik, Universität Bielefeld, D-4800 Bielefeld 1, Federal Republic of Germany
}

Received 3 August 1992, in final form 9 October 1992

\begin{abstract}
We measured the total ionization asymmetry $A$ in dependence on the incident electron energy $E$. Here 'total' refers to integration over all emission angles and energy partitions of the outgoing electrons. From a threshold value of $A=0.125$ the $A(E)$ curve rises smoothly toward a broad maximum of $A_{\max }=0.31$ at $E_{\max }=8.3 \mathrm{eV}$. The fall-off towards higher energies is quite similar to that of the other one-electron atoms. However, it shows a structure which can be explained by contributions from autoionizing $\mathbf{P}$ states. Bartschat has made a theoretical estimate of $A(E)$ for various atoms. At low energies the agreement with our data is satisfactory, at higher energies the experimental $A$ values are considerably smaller than the theoretical ones. In the threshold region measurements were performed with small electron energy width $(0.1 \mathrm{eV})$. No structure in the $A(E)$ curve was found. The slope at threshold was determined as $\mathrm{dA} / \mathrm{d} E=(0.136 \pm 0.005) \mathrm{eV}^{-1}$.
\end{abstract}

\section{Introduction}

The spin asymmetry $\boldsymbol{A}$ is determined according to

$$
A=\frac{1}{P_{\mathrm{e}} P_{\mathrm{a}}} \frac{N^{\downarrow \uparrow}-N^{\uparrow \uparrow}}{N^{\downarrow \uparrow}+N^{\uparrow \uparrow}}
$$

where $N^{\downarrow \uparrow}$ and $N^{\uparrow \uparrow}$ are the ion signals obtained with antiparallel and parallel beam polarizations, respectively, in the two beams. $P_{\mathrm{e}}$ is the electron polarization and $P_{\mathrm{a}}$ is the atomic polarization referring to the spin-polarized valence electron of the Cs atom. The nuclear polarization of the Cs atom is irrelevant here.

Up to now spin-asymmetry measurements in total ionization have been made for the one-electron atoms $\mathrm{H}, \mathrm{Li}, \mathrm{Na}, \mathrm{K}$ and the two-electron atom $\mathrm{He}\left(2^{3} \mathrm{~S}\right.$ ) (references in table 1). Common to all results is

$$
A \geqslant 0
$$

at all energies. The investigated atoms differ distinctly in their $A(E)$ curves: The slope of $A(E)$ at threshold is nearly zero for $\mathrm{Li}, \mathrm{Na}$ and $\mathrm{K}$, slightly positive for $\mathrm{He}\left(2^{3} \mathrm{~S}\right)$, significantly positive for $\mathrm{H}$; only for $\mathrm{K}$ is the shape of $A(E)$ irregular exhibiting a minimum between threshold and maximum. Therefore, one reason for studying $\mathrm{Cs}$ was to find out whether a trend exists which leads to very different $A(E)$ curves for the heavy alkali atoms. 
Table 1. Comparison of $A$ for different atoms.

\begin{tabular}{llll}
\hline Atom & $A$ at $E_{1}$ & $A_{\max }$ at $E_{\max }$ & $E_{\max } / E_{\mathrm{I}}$ \\
\hline $\mathrm{H}^{\mathrm{a}}$ & $0.43 \pm 0.04$ & $0.49 \neq 0.04$ & 1.26 \\
$\mathrm{He}\left(2^{3} \mathrm{~S}\right)^{\mathrm{b}}$ & $0.34 \pm 0.03$ & $0.42 \pm 0.01$ & 1.54 \\
$\mathrm{Li}^{\mathrm{c}}$ & $0.43 \pm 0.01$ & $0.49 \pm 0.01$ & 1.77 \\
$\mathrm{Na}^{\mathrm{c}}$ & $0.45 \pm 0.03$ & $0.46 \pm 0.02$ & 1.55 \\
$\mathrm{~K}^{\mathrm{c}}$ & $0.25 \pm 0.02$ & $0.28 \pm 0.01$ & 3.46 \\
$\mathrm{Cs}^{\mathrm{d}}$ & $0.13 \pm 0.01$ & $0.31 \pm 0.01$ & 2.26 \\
\hline
\end{tabular}

- Fletcher et al (1985),

Baum et al (1989).

'Baum et al (1985).

'This work.

\section{Experiment}

In a crossed-beam arrangement (figure 1) we intersected a polarized electron beam from a $\mathrm{GaAs}$ source with a polarized $\mathrm{Cs}$ atomic beam. The caesium atomic beam had a spin polarization close to unity and was produced by employing a recirculating oven and optical pumping with two laser diodes (Baum et al 1991).

The electron current in the interaction region was typically $0.1 \mu \mathrm{A}$, the electron polarization $P_{\mathrm{c}} \approx 0.3$. For the first measurements extended over a wide energy range, the energy width of the electron beam, emitted from the cathode with $\Delta E \approx 0.45 \mathrm{eV}$, was not reduced by the $180^{\circ}$ spherical monochromator. Later, for the measurements in the threshold region, the monochromator was tuned to reduce the energy width to $\Delta E \approx 0.1 \mathrm{eV}$. This value was calculated from the voltage settings and is consistent with results of retarding-potential measurements.

The electron polarization was determined with a small-size retarding-field Mott polarimeter (Rott 1991). The polarimeter was constructed by slightly modifying a

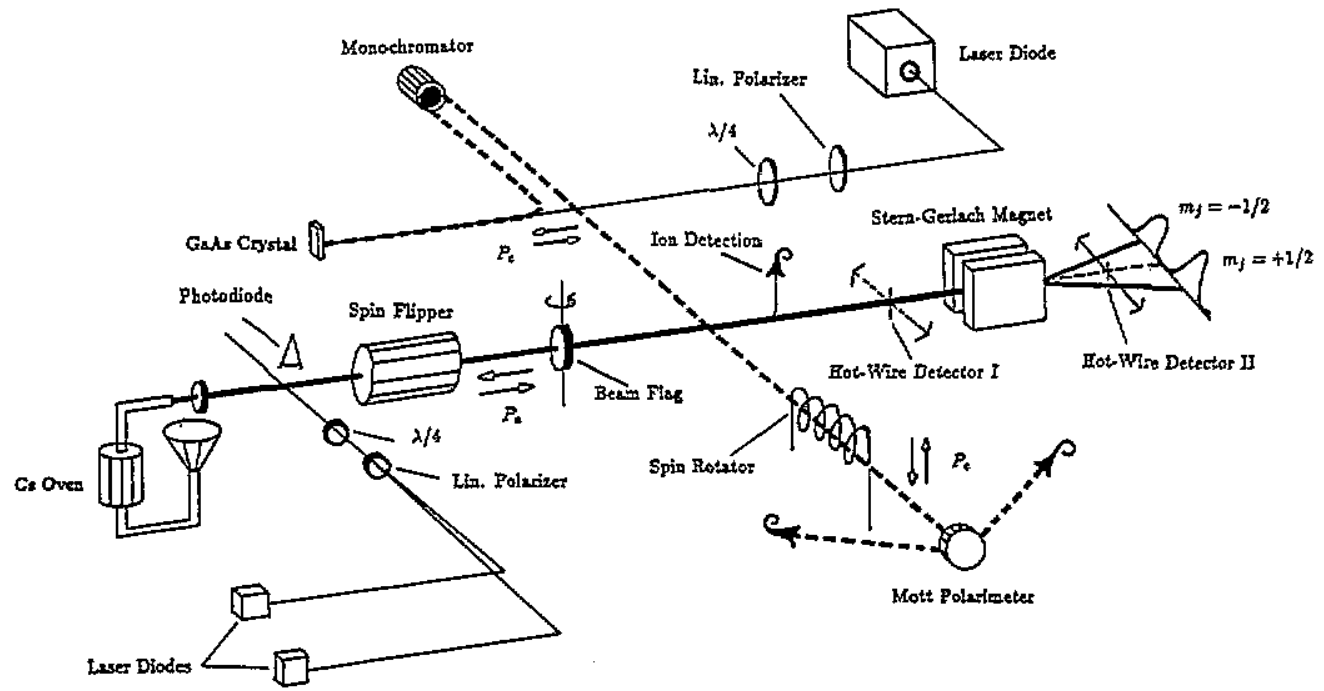

Figure 1. Experimental arrangement. 
design of Gellrich and Kessler (1991). By using a thick thorium foil we obtained an analysing power of $S_{\text {eff }}=0.4$ at $45 \mathrm{keV}$ electron scattering energy.

The atomic beam had a density of $10^{9}$ atoms $/ \mathrm{cm}^{3}$ and a diameter of about $0.5 \mathrm{~cm}$ in the interaction region. The atomic polarization $P_{\mathrm{a}}$ was measured with an analysing Stern-Gerlach magnet, as described earlier (Baum et al 1991), yielding values of $P_{\mathrm{a}}>0.9$. To guide the atomic spins, a magnetic field of about $5 \times 10^{-6} \mathrm{~T}$, collinear with the atomic beam, was present in the interaction region.

The produced ions were extracted from the interaction region with a small electric field of about $0.5 \mathrm{~V} \mathrm{~cm}^{-1}$ oriented in the atomic beam direction. After passage through a grid the ions were deflected in a cylindrical condenser and directed towards a Channeltron multiplier for detection. On the first plateau of the total ionization cross section $(E \approx 10 \mathrm{eV})$ the ion counting rate was typically $5000 \mathrm{~s}^{-1}$. For obtaining the spin asymmetry according to equation (1) ion rates were observed at each energy with parallel and antiparallel spin orientations of the two beams. The orientations were changed by using the spin flipper in the atomic beam line. Measurements were also made with different settings of the quarter-wave plates which determine the circular light polarization for the electron source, as well as those for the optical pumping of the atomic beam. Above $20 \mathrm{eV}$ background events contributed appreciably to the rate. They were measured separately (atomic beam flag closed) and subtracted accordingly. The data accumulation time for one data point was typically 600 to $1000 \mathrm{~s}$.

\section{Results}

Figure 2 shows measured $A$ values over a wide range of $E / E_{1}$ where $E_{\mathrm{r}}$ is the ionization energy. In the threshold region $\left(1<E / E_{\sharp}<2\right)$ we plotted every third of our closely

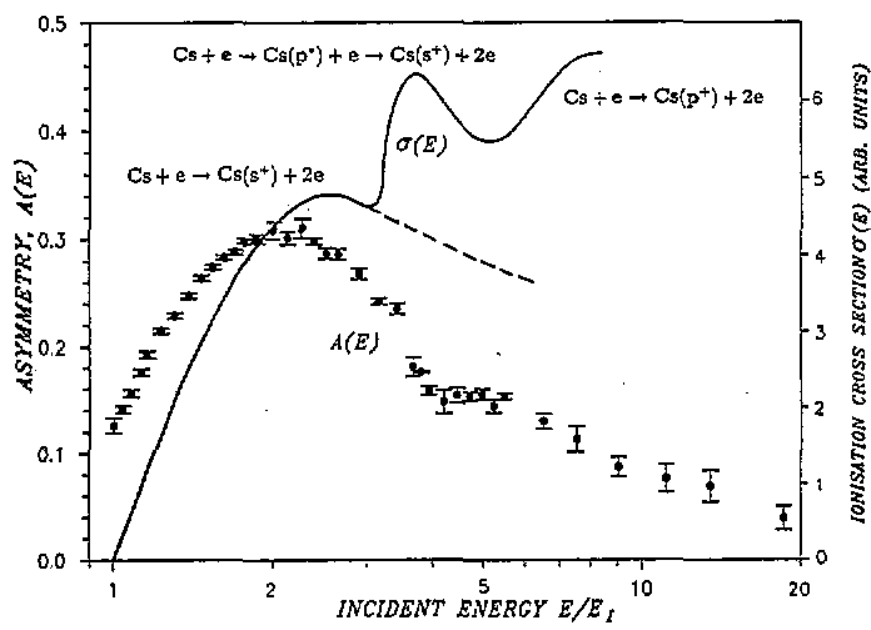

Figure 2. Ionization asymmetry $A$ (our data points/left scale) and ionization cross section $\sigma$ (full curve/right scale) in their dependence on the incident-electron energy $E$ (in units of $\left.E_{1}(\mathrm{Cs})=3.89 \mathrm{eV}\right)$. The cross section information was taken from Zapasochnyi and Aleksakhin (1969). The broken curve indicates the part of the cross section which is due to direct $\mathrm{s}$ electron ionization. The superscripts have the following meaning: *, electron excitation; + , electron vacancy. 
spaced high-resolution data points which are discussed in detail later on. The fall-off on the high-energy side of the maximum has two causes. Firstly, this shows the expected general behaviour of $A(E)$ for the ionization of the polarized s electrons. Secondly, events from the ionization of the unpolarized p electrons, which sets in at about $17 \mathrm{eV}$, also contribute to the fall-off. Furthermore, around $15 \mathrm{eV}$ autoionization from excited $P$ states causes a peak in the cross section curve (cf figure 2) and is thought to be responsible for the shoulder in the $A(E)$ curve.

There are no theoretical predictions for $A(E)$ of $C$ s available yet. In table 1 we list the values of the asymmetry at threshold and at the maximum for all the atoms studied thus far. Only for $\mathrm{He}\left(2^{3} \mathrm{~S}\right)$ and $\mathrm{Cs}$ do the threshold values lie substantially below the maximum.

The value of $A$ is directly related to the ratio $r$ of singlet to triplet cross section by

$$
\begin{array}{ll}
\sigma^{\uparrow \uparrow}=\sigma_{\mathrm{T}} & \sigma^{\downarrow \uparrow}=\frac{1}{2} \sigma_{\mathrm{S}}+\frac{1}{2} \sigma_{\mathrm{T}} \\
A=\frac{\sigma_{\mathrm{S}}-\sigma_{\mathrm{T}}}{\sigma_{\mathrm{S}}+3 \sigma_{\mathrm{T}}} & r=\frac{\sigma_{\mathrm{S}}}{\sigma_{\mathrm{T}}}=\frac{1+3 A}{1-A} .
\end{array}
$$

Consequently, $A=1$ corresponds to pure singlet scattering and $A=-\frac{1}{3}$ to pure triplet scattering. The observed threshold value of $A=0.13$ leads to $r=1.60$, giving evidence for a strong triplet component right at threshold. The positive slope of $A(E)$ near threshold is interesting as it shows a decrease of triplet scattering with increasing energy. This behaviour was also observed in $\mathrm{He}\left(2^{3} \mathrm{~S}\right)$ (Baum et al 1989) and discussed in more detail there. The distorted-wave Born approximation (DWBA) of Bartschat (1990) for $\mathrm{He}\left(2^{3} \mathrm{~S}\right)$ gives a positive slope of $A(E)$ near threshold. However, from the calculation one cannot extract which mechanisms are responsible for this behaviour (Bartschat 1992). For Cs the region of positive slope is quite extended in energy (3 eV).

In figure 3 we show Bartschat's calculations for $\mathrm{Li}$ and $\mathrm{K}$. Since these and also his results for $\mathrm{He}\left(2^{3} \mathrm{~S}\right)$ and $\mathrm{Na}$ all lie very close together, it can be expected that the same 'maximum interference' distorted-wave Born approximation (DWBA) would yield a

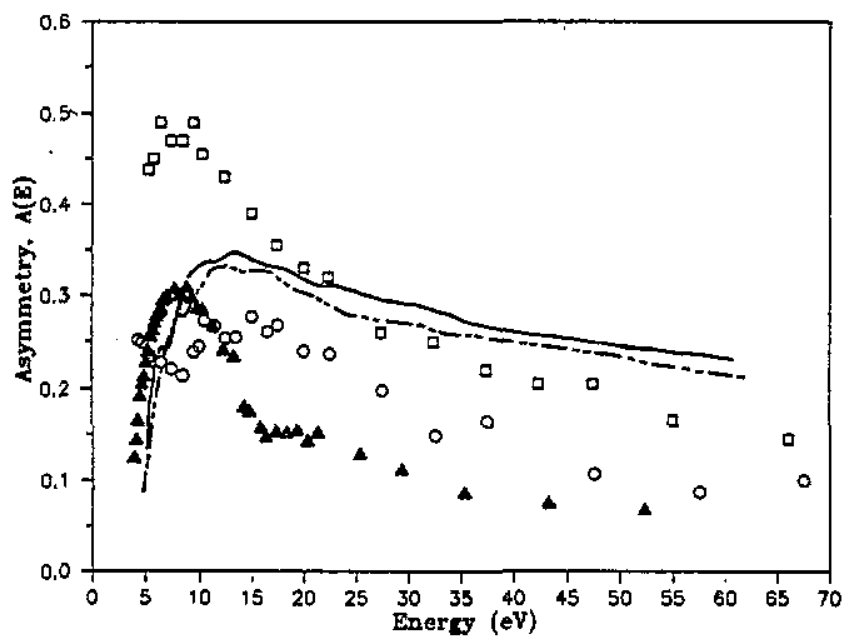

Figure 3. Ionization asymmetry $A$ against electron energy $E$ (linear scale). The upper curve gives the theoretical results of Bartschat (1990) for $\mathrm{Li}$, the lower curve, for $\mathrm{K}$. The symbols refer to experimental results: $\mathrm{Li}$, open squares; $\mathrm{K}$, triangles; $\mathrm{Cs}$, full triangles (Baum et al 1985 for $\mathrm{Li}$ and $\mathrm{K}$, this work for $\mathrm{Cs}$ ). 
similar $A(E)$ curve for Cs, not taking spin-orbit coupling and autoionization effects into account. Figure 3 also shows the experimental results for $\mathrm{Li}, \mathrm{K}$ and $\mathrm{Cs}$. For $\mathrm{Li}$ (as well as for $\mathrm{H}, \mathrm{Na}$ and $\mathrm{He}\left({ }^{3} \mathrm{~S}\right)$, not shown in figure 3 ) the experimental $A$-values at threshold and around the maximum of $A(E)$ are significantly higher than the theoretical results and the slope of the fall-off towards higher energies is steeper. For $\mathrm{K}$, the experimental data show an irregular energy dependence, whereas the shape of the $A(E)$ curve for $C$ s does not follow this trend and is more similar to those of the other atoms studied. Since for Cs the p-electron ionization contributes on the highenergy side of the maximum, a more rapid decrease of $A(E)$ than predicted by theory is not surprising.

The $A(E)$ data obtained with $\Delta E \approx 0.1 \mathrm{eV}$ are shown in figure 4. The data points lie on a smooth curve. They do not exhibit 'undulations' as predicted by Temkin (1966, 1982 ) and possibly seen in $A(E)$ for atomic hydrogen (Guo et al 1990). Kelley et al (1983) performed high-resolution measurements on $\mathrm{Na}$ and did not see any structure either.

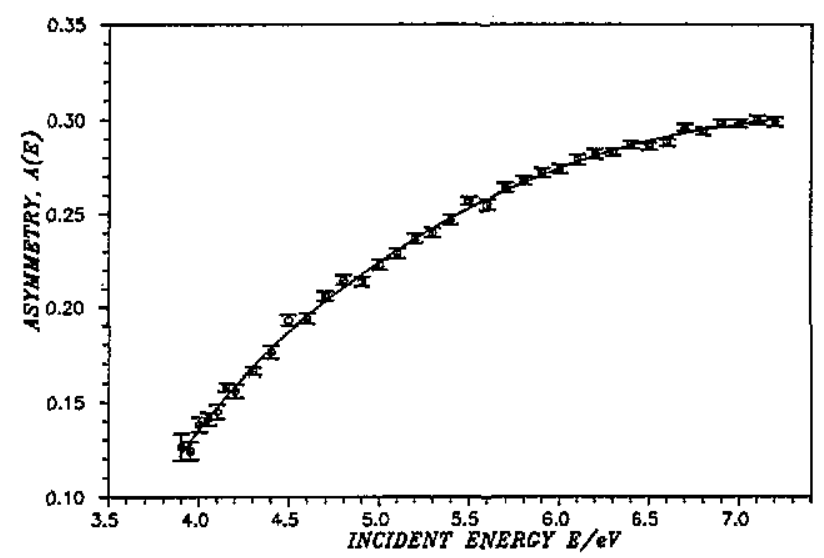

Figure 4. Measurements of $A(E)$ near threshold with improved energy resolution. The full curve is the best fit described in the text.

We searched for the presence of non-statistical structures in the data by first fitting the asymmetry measurements with a six-parameter polynomial in powers of the incident energy. The resulting reduced chi-square, 1.17 for 31 degrees of freedom (confidence level $23 \%$ compared to a norm of $50 \%$ ), while slightly short of the ideal, is nonetheless sufficient for our purposes. We then calculated the parameter $d$ of the Durbin-Watson statistics (Durbin and Watson 1950, 1951), according to the prescription $d=$ $\Sigma_{i=1}^{N}\left(e_{i}-e_{i-1}\right)^{2} / \Sigma_{i=1}^{N} e_{i}^{2}$, where $e_{i}$ is the normalized residual, $i$ is the order parameter and $N=37$ is the number of data points. The resulting value of $d=2.48$ is so much larger than the upper limit of 1.53 for $5 \%$ significance, that we can safely rule out any non-random ordering of the residuals and hence any presence of structure at the statistical level of sensitivity of the experiment.

From the data of figure 4 we determined the slope of $A(E)$ at threshold as

$$
\left(\frac{\mathrm{d} A}{\mathrm{~d} E}\right)_{E=E_{1}}=(0.136 \pm 0.005) \mathrm{eV}^{-1} \text {. }
$$

This might be of interest for the theory of threshold behaviour which is not yet available for Cs. 


\section{Acknowledgments}

Stimulating discussions with Professor M S Lubell, (CC of CUNY, New York City) are gratefully acknowledged. This work has been supported by the Deutsche Forschungsgemeinschaft in SFB 216 and by the University of Bielefeld.

\section{References}

Bartschat K 1990 J. Phys. B: At. Mol. Opt. Phys. 23981

1992 Private communication

Baum G, Fink M, Raith W, Steidl H and Taborski J 1989 Phys. Rev. A 406734

Baum G, Granitza B, Hesse S, Leuer B, Raith W, Rott K, Tondera M and Witthuhn B 1991 Z. Phys. D 22 431

Baum G, Moede M, Raith W and Schröder W 1985 J. Phys. B: Al. Mol. Phys. 18531

Durbin J and Watson G S 1950 Biometrika 37409

- 1951 Biometrika 38159

Fletcher G D, Alguard M J, Gay T J, Hughes V W, Wainwright P F, Lubell M S and Raith W 1985 Phys. Rev. A 312854

Gellrich A and Kessler J 1991 Phys. Rev. A 43204

Guo X Q, Crowe D M, Lubel M S, Tang F C, Vasilakis K, Slevin J and Eminyan M 1990 Phys. Rev. Lett. 651857

Johnson J 1972 Econometric Methods 2nd edn (New York: McGraw-Hill)

Kelley M H, Rogers W T, Celotta R I and Mielczarek S R 1983 Phys. Rev. Lett. 512191

Rott K 1991 Diplomarbeit Universität Bielefeld

Temkin A 1966 Phy's. Rev. Lett. 16835

—_ 1982 Phys. Rev. Lett. 49365

Zapesochnyi I P and Alekasakhin I S 1969 Sov. Phys.-JETP 28 41-5 